\title{
Effective Nullstellensatz and geometric degree for zero-dimensional ideals
}

by

\section{A. Fabiano (Arcavacata di Rende), G. Pucci (Arcavacata di Rende) and A. YGer (Talence)}

1. Introduction. Let $F:=\left(F_{1}, \ldots, F_{k}\right): \mathbb{C}^{n} \rightarrow \mathbb{C}^{k}$ be a polynomial map with

$$
\operatorname{deg} F_{2} \geq \operatorname{deg} F_{3} \geq \ldots \geq \operatorname{deg} F_{k} \geq \operatorname{deg} F_{1}>0
$$

and $G$ a polynomial in $n$ variables such that $G \mid F^{-1}(0) \equiv 0$. The Hilbert Nullstellensatz guarantees the existence of polynomials $A_{1}, \ldots, A_{k}$ such that

$$
G^{s}=A_{1} F_{1}+\ldots+A_{k} F_{k}
$$

where $s$ is an integer $\geq 0$. In the usual proofs of this result, one is not concerned with estimates about the degree of the $A_{j}$ 's and the exponent $s$. This question was considered by Brownawell [Br1] and later by N. Fitchas [Fi] and Kollár $[\mathrm{K}]$. Kollár got the best estimates under the technical hypothesis $\operatorname{deg} F_{j} \neq 2$ for $j>1$. Namely, it is possible to solve (1.1) with the estimates

$$
\left\{\begin{array}{l}
\max \left(\operatorname{deg} A_{j} F_{j}\right) \leq(1+\operatorname{deg} G) \prod_{j=1}^{\min (k, n)} \operatorname{deg} F_{j}, \\
s \leq \prod_{j=1}^{\min (k, n)} \operatorname{deg} F_{j} .
\end{array}\right.
$$

The result of Brownawell-Kollár is in fact a result about homogeneous ideals which has also an interpretation in algebraic geometry. This was pointed out by Brownawell [Br2] (see also [T]) as follows:

Let $\mathcal{P}_{1}, \ldots, \mathcal{P}_{m}$ be the isolated prime components of the homogeneous ideal $\mathcal{U}:=\left({ }^{\mathrm{h}} F_{1}, \ldots,{ }^{\mathrm{h}} F_{k}\right)$ where ${ }^{\mathrm{h}} F$ is the homogenized version of $F$. Then, if $\operatorname{deg} F_{i} \neq 2$ for $i>1$, there exist integers $e_{1}, \ldots, e_{m}$ (depending on the decomposition of $\mathcal{U}$ ) such that

$$
\sum_{i=1}^{m} e_{i} \leq \prod_{j=1}^{\min (k, n)} \operatorname{deg} F_{j}, \quad \mathcal{P}_{1}^{e_{1}} \cap \ldots \cap \mathcal{P}_{m}^{e_{m}} \subset \mathcal{U}
$$


If $G \equiv 0$ on $F^{-1}(0)$, then ${ }^{\mathrm{h}} G \in \mathcal{P}_{1} \cap \ldots \cap \mathcal{P}_{q}$, where $\mathcal{P}_{1}, \ldots, \mathcal{P}_{q}$ are the isolated prime components which do not contain $X_{0}$; it follows from (1.3) that, if

then

$$
s_{1}:=\sum_{i=1}^{q} e_{i}, \quad s_{2}:=\sum_{i=q+1}^{m} e_{i},
$$

$$
\left({ }^{\mathrm{h}} G\right)^{s_{1}} X_{0}^{s_{2}} \in\left(\mathcal{P}_{1}^{e_{1}} \cap \ldots \cap \mathcal{P}_{q}^{e_{q}}\right) \cap\left(\mathcal{P}_{q+1}^{e_{q+1}} \cap \ldots \cap \mathcal{P}_{m}^{e_{m}}\right) \subset \mathcal{U} .
$$

This implies, if one restricts the situation to $X_{0}=1$,

$$
\left\{\begin{array}{l}
G^{s_{1}}=\sum_{j=1}^{k} A_{j} F_{j} \\
\operatorname{deg} A_{j} F_{j} \leq s_{1} \operatorname{deg} G+s_{2} \leq\left(s_{1}+s_{2}\right) \max (\operatorname{deg} G, 1) \\
\quad \leq \max (\operatorname{deg} G, 1) \prod_{j=1}^{\min (k, n)} \operatorname{deg} F_{j} .
\end{array}\right.
$$

Note that the value of the exponent $s$ in (1.1) (namely $s=s_{1}+s_{2}$ ) which is provided that way depends on the decomposition of the homogeneous ideal.

On the other hand, in the particular case where $\# F^{-1}(0)<\infty$, there is another version of the Nullstellensatz following the work of M. Noether. For any $\alpha \in F^{-1}(0)$, let

$$
\nu_{\alpha}(F):=\min \left\{p \in \mathbb{N},\left(\sqrt{F_{1} \mathcal{O}_{\alpha}+\ldots+F_{k} \mathcal{O}_{\alpha}}\right)^{p} \subset F_{1} \mathcal{O}_{\alpha}+\ldots+F_{k} \mathcal{O}_{\alpha}\right\},
$$

that is, the local Noether exponent of the map $F$ at $\alpha$. It is well known [GH] that

$$
\nu_{\alpha}(F) \leq \mu_{\alpha}(F):=\operatorname{dim}_{\mathbb{C}}\left(\frac{\mathcal{O}_{\alpha}}{F_{1} \mathcal{O}_{\alpha}+\ldots+F_{k} \mathcal{O}_{\alpha}}\right) .
$$

If $G \equiv 0$ on $F^{-1}(0)$, then

$$
G^{\nu} \in\left(F_{1}, \ldots, F_{k}\right)
$$

where

$$
\nu:=\max _{\alpha \in F^{-1}(0)} \nu_{\alpha}(F)
$$

Nevertheless, this result, which provides an exponent

$$
\nu=\max _{\alpha \in F^{-1}(0)} \nu_{\alpha}(F) \leq \max _{\alpha \in F^{-1}(0)} \mu_{\alpha}(F)
$$

depending only on the ideal $\left(F_{1}, \ldots, F_{k}\right)$, does not give any information on the degree of the $A_{j} F_{j}$ 's where

$$
G^{\nu}=\sum_{j=1}^{k} A_{j} F_{j} .
$$


The main objective of this paper is, under the hypothesis $\# F^{-1}(0)<\infty$, to find some balance between good estimates for $s$ (that is, estimates depending on the affine situation and not on the projective one) as in (1.6) and good control on the degree of the $A_{j} F_{j}$ 's such as in (1.4).

In order to do that, we consider two different approaches.

- The first one works only if $k=n, \# F^{-1}(0)<\infty$, and the map $\left(F_{1}, \ldots, F_{n}\right)$ is dominant (that is, $\left[\mathbb{C}\left(X_{1}, \ldots, X_{n}\right): \mathbb{C}\left(F_{1}, \ldots, F_{n}\right)\right]<\infty$, or also $F_{1}, \ldots, F_{n}$ are algebraically independent over $\left.\mathbb{C}\right)$. It uses essentially two ingredients.

Primo, some variants (which will be detailed in Section 2) of the fact that for any $G \in \mathbb{C}[X]$, the map

$$
R_{F, G}: w \mapsto\left[\begin{array}{c}
G d X_{1} \wedge \ldots \wedge d X_{n} \\
F_{1}-w_{1}, \ldots, F_{n}-w_{n}
\end{array}\right]
$$

(where [ ] denotes the total sum of residues in the sense of Grothendieck) is a rational map.

Secondo, some very classical combinatory argument due to Perron $[\mathrm{Pe}]$, and which is also a basic tool in the work of Jelonek, Płoski and P. CassouNoguès ([Je], [CN], [CNPł], [Pł1], [Pł2]). This first approach allows us to deal with three particular situations:

(a) $\left(F_{1}, \ldots, F_{n}\right)$ is a proper map;

(b) $\left(F_{1}, \ldots, F_{n}\right)$ is a proper map over the origin;

(c) $\left(F_{1}, \ldots, F_{n}\right)$ satisfies some "separation condition" (as in [PłT]) over the origin.

(a) In case (a), $R_{F, G}$ is in fact a polynomial, with degree controled by the Łojasiewicz exponent $\delta>0$ of the map $\left(F_{1}, \ldots, F_{n}\right)$, defined as

$$
\delta:=\min \left\{r>0: \liminf _{\|\zeta\| \rightarrow \infty}\|F(\zeta)\| /\|\zeta\|^{r}\right\}>0 .
$$

There are in this subcase two different results; either one can profit from the knowledge of $\delta$ and get, using the Cauchy-Weil formula, that if $G \mid F^{-1}(0) \equiv$ 0 , then, if $D:=\max _{1 \leq j \leq n}\left(\operatorname{deg} F_{j}\right), \nu:=\max _{\alpha \in F^{-1}(0)} \nu_{\alpha}(F)$,

$$
\left\{\begin{array}{l}
G^{\nu}=\sum_{j=1}^{k} A_{j} F_{j}, \\
\operatorname{deg} A_{j} F_{j} \leq D\left(\mathrm{E}\left(\frac{2}{\delta}\left(\sum_{j=1}^{n} \operatorname{deg} F_{j}+\nu \operatorname{deg} G\right)\right)-n\right)
\end{array}\right.
$$

(where $\mathrm{E}(\cdot)$ denotes the integral part); or one can essentially reinterpret some algebraic method (already introduced in $[\mathrm{CN}],[\mathrm{CNP}]]$ ), which gives, for some $s \leq d$ (where $d$ is the geometric affine degree $d:=\left[\mathbb{C}\left(X_{1}, \ldots, X_{n}\right)\right.$ : 
$\left.\mathbb{C}\left(F_{1}, \ldots, F_{n}\right)\right]$ of the map $\left.F\right)$,

$$
G^{s}=\sum_{j=1}^{n} A_{j} F_{j}, \quad \operatorname{deg} A_{j} F_{j} \leq \operatorname{deg} G \prod_{j=1}^{n} \operatorname{deg} F_{j} .
$$

Such estimates were obtained by P. Cassou-Noguès in [CN]. One can compare these estimates with (1.7) only if one has some estimate from below for the Eojasiewicz exponent.

(b) In case (b), the method that leads to (1.8) works exactly the same and provides exactly the same result (already obtained in [CNP1]). The main difference with $[\mathrm{CNPl}]$ is that here we can extend the method to some particular situation when the map is not proper at the origin, namely in case (c).

(c) In this case, if $G \mid F^{-1}(0) \equiv 0$, then, for some $s \leq d$ ( $d$ being again the geometric degree of $F$ ),

$$
G^{s}=\sum_{j=1}^{n} A_{j} F_{j}, \quad \operatorname{deg} A_{j} F_{j} \leq n(\operatorname{deg} G+1)\left(\operatorname{deg} F_{1} \cdot \ldots \cdot \operatorname{deg} F_{n}+d\right) .
$$

- The second approach works in the general case where $\# F^{-1}(0)<\infty$, but it profits from the knowledge of the Łojasiewicz exponent $q$ at infinity of the map $\left(F_{1}, \ldots, F_{k}\right)$; since the case of proper maps has already been treated, this approach is interesting in the case when $q \leq 0$. Note that, from Brownawell [Br1], we know that

$$
q \geq 1-(n-1) \prod_{j=1}^{\min (k, n)} \operatorname{deg} F_{j} .
$$

One just interprets the condition

$$
\|F(\zeta)\| \geq \kappa\|\zeta\|^{q}, \quad\|\zeta\| \gg 1
$$

as a condition on the map $\left({ }^{\mathrm{h}} F_{1}, \ldots,{ }^{\mathrm{h}} F_{k}\right)$ on the unit sphere $S^{2 n+1}$. The key algebraic ingredient is the Briançon-Skoda theorem about integral closures of ideals ([BS], [LT]); if $G \mid F^{-1}(0) \equiv 0$, then

$$
G^{\gamma \nu}=\sum_{j=1}^{k} A_{j} F_{j}, \quad \operatorname{deg} A_{j} F_{j} \leq \gamma(\nu \operatorname{deg} G+D+\max (-q, 0)),
$$

with

$$
\gamma:=\min (n+1, k), \quad \nu:=\max _{\alpha \in F^{-1}(0)} \nu_{\alpha}(F), \quad D:=\max _{1 \leq j \leq k}\left(\operatorname{deg} F_{j}\right) .
$$

Moreover, this result can be improved if one assumes that the maximal ideal $\left(X_{0}, \ldots, X_{n}\right)$ is not an embedded prime component in the decomposition of $\left({ }^{\mathrm{h}} F_{1}, \ldots,{ }^{\mathrm{h}} F_{k}\right.$ ) (or the depth of ${ }^{\mathrm{h}} F_{1 n+1} \mathcal{O}_{0}+\ldots+{ }^{\mathrm{h}} F_{k n+1} \mathcal{O}_{0}$ is $\geq 2$ ); in this 
case, if $G \mid F^{-1}(0) \equiv 0$, then

$$
G^{\nu}=\sum_{j=1}^{k} A_{j} F_{j}, \quad \operatorname{deg} A_{j} F_{j} \leq \nu \operatorname{deg} G+n[D+\max (-q, 0)] .
$$

Note that such results englobe the result obtained in [CNPł] for "stably inconsistent maps" in the sense of $[\mathrm{Fi}]\left(\#\left\{F^{-1}(w)\right\}=0\right.$ for $\left.\|w\| \ll 1\right)$.

In order to be able to compare the different versions of the Nullstellensatz which we propose here, it is of course necessary to remark that estimates (1.7), (1.11), (1.12) have the advantage of being much more precise than (1.8) or (1.9), but need some a priori information about the Eojasiewicz exponent ( $\delta$ in the proper case, $q$ in the general case). Note that, if the map $\left(F_{1}, \ldots, F_{n}\right)$ is proper at 0 , and if $G \mid F^{-1}(0) \equiv 0$, then one has from (1.11),

$$
G^{(n+1) \nu}=\sum_{j=1}^{n} A_{j} F_{j}, \quad \operatorname{deg} A_{j} F_{j} \leq(n+1)(\nu \operatorname{deg} G+D),
$$

which seems clearly in general better than estimates (1.8); this shows the real power of the Briançon-Skoda theorem. We will see that, though the first approach does not seem to lead to such estimates, it has the advantage of being completely constructive (in terms of computations of total sums of residues). Another interesting remark is that the methods in Section 3 provide some effective Nullstellensätze independently of the results of Brownawell. Such methods could be a starting point to get some complete analytic proof of the Brownawell-Kollár results. Note also that all the results here have been obtained in the case when the zero set of the entries is discrete. This is a rather good situation for effectivity problems, since one knows in this particular case that the Buchberger algorithm (to get a standard basis for the ideal) runs in subexponential time (see [CoS]). The search for estimates depending on the affine degree instead of the projective one in such effectivity problems, dealing with the non-discrete situation, has recently been investigated in [HGi].

Ackowledgements. Part of this work was inspired by discussions with A. Płoski and by a lecture he gave in Bordeaux as an invited Professor in March 1995. We also would like to thank Pierrette Cassou-Noguès who suggested in $[\mathrm{CN}]$ this kind of problems in relation with [BGVY]. This paper was written while the third author was invited to the University of Calabria; he would like to take here the opportunity to thank warmly this institution.

2. Newton sums and total sums of residues. Let $f=\left(f_{1}, \ldots, f_{n}\right)$ be a polynomial map from $\mathbb{C}^{n}$ to $\mathbb{C}^{n}$ such that $\# f^{-1}(0)<\infty$. For any $r \in \mathbb{C}\left(X_{1}, \ldots, X_{n}\right)$ with no poles on $V=f^{-1}(0)$, one can define the total 
sum of residues of $r$ with respect to $f$ as

$$
\begin{aligned}
& {\left[\begin{array}{c}
r d X_{1} \wedge \ldots \wedge d X_{n} \\
f_{1}, \ldots, f_{n}
\end{array}\right]:=\lim _{\varepsilon \rightarrow 0} \frac{(-1)^{n(n-1) / 2}(n-1) !}{(2 i \pi)^{n}}} \\
& \quad \times \sum_{\alpha \in V} \int_{\|\zeta-\alpha\|=\varepsilon} \frac{\sum_{j=1}^{n}(-1)^{j-1} \overline{f_{j}(\zeta)} \bigwedge_{l \neq j} \overline{d f_{l}(\zeta)} \wedge r(\zeta) d \zeta}{\|f(\zeta)\|^{2 n}}
\end{aligned}
$$

If $r, f_{1}, \ldots, f_{n}$ belong respectively to $\mathbb{F}\left(X_{1}, \ldots, X_{n}\right), \mathbb{F}\left[X_{1}, \ldots, X_{n}\right]$, where $\mathbb{F}$ is a subfield of $\mathbb{C}$, then the total sum of residues of $r$ with respect to $f$ is also in $\mathbb{F}$. If $J(f)$ denotes the Jacobian of the map $\left(f_{1}, \ldots, f_{n}\right)$, then

$$
\left[\begin{array}{c}
r J(f) d X_{1} \wedge \ldots \wedge d X_{n} \\
f_{1}, \ldots, f_{n}
\end{array}\right]=\sum_{\alpha \in V} \mu_{f}(\alpha) r(\alpha)
$$

where $\mu_{f}(\alpha)$ is the local multiplicity of the map $f$ at $\alpha$. In particular,

$$
\left[\begin{array}{c}
r J(f) d X_{1} \wedge \ldots \wedge d X_{n} \\
f_{1}, \ldots, f_{n}
\end{array}\right]
$$

can be interpreted as the trace of the multiplication operator

$$
\bar{r}: \mathbb{C}[X] /\left(f_{1}, \ldots, f_{n}\right) \rightarrow \mathbb{C}[X] /\left(f_{1}, \ldots, f_{n}\right)
$$

defined (if $r=r_{1} / r_{2}$ ), as $\bar{r}=\bar{r}_{1} \cdot \bar{r}_{2}^{-1}$, where

$$
\bar{r}_{1}: \bar{g} \mapsto \overline{g r}_{1}, \quad \bar{r}_{2}: \bar{g} \mapsto \overline{g r}_{2} .
$$

Moreover, if

$$
d:=\operatorname{dim}_{\mathbb{C}} \mathbb{C}[X] /\left(f_{1}, \ldots, f_{n}\right),
$$

then the symmetric functions of the collection $\{r(\alpha): \alpha \in V\}, \sigma_{1}, \ldots, \sigma_{d}$, can be computed in terms of the Newton sums $S_{1}, \ldots, S_{d}$ of these numbers, namely

$$
S_{j}=\left[\begin{array}{c}
r^{j} J(f) d X_{1} \wedge \ldots \wedge d X_{n} \\
f_{1}, \ldots, f_{n}
\end{array}\right]
$$

Then, the characteristic polynomial of the multiplication operator $\bar{r}$,

$$
X^{d}-\sigma_{1} X^{d-1}+\ldots+(-1)^{d} \sigma_{d}
$$

can be expressed in terms of total sums of residues modulo the Newton relations.

Let now $F=\left(F_{1}, \ldots, F_{n}\right)$ be a dominant polynomial map from $\mathbb{C}^{n}$ to $\mathbb{C}^{n}$, i.e. a polynomial map such that $\left[\mathbb{C}\left(X_{1}, \ldots, X_{n}\right): \mathbb{C}\left(F_{1}, \ldots, F_{n}\right)\right]<\infty$. We have the following:

LEMma 2.1. There exists some algebraic hypersurface $\Sigma$ in $\mathbb{C}^{n}$ such that, for any $w \in \mathbb{C}^{n} \backslash \Sigma, \#\left\{\zeta: F_{j}(\zeta)=w_{j}, j=1, \ldots, n\right\}<\infty$ and, for any 
$G \in \mathbb{C}\left[X_{1}, \ldots, X_{n}\right]$, there exists $R_{F, G} \in \mathbb{C}\left(X_{1}, \ldots, X_{n}\right)$, with poles on $\Sigma$, such that, for any $w \in \mathbb{C}^{n} \backslash \Sigma$,

$$
\left[\begin{array}{c}
G d X_{1} \wedge \ldots \wedge d X_{n} \\
F_{1}-w_{1}, \ldots, F_{n}-w_{n}
\end{array}\right]=R_{F, G}(w)
$$

Proof. For any $j \in\{1, \ldots, n\}$, there are $N_{j} \in \mathbb{N}^{*}$ and $A_{j, 0}, \ldots, A_{j, N_{j}} \in$ $\mathbb{C}\left[X_{1}, \ldots, X_{n}\right]$ such that

$$
A_{j, 0}(F) X_{j}^{N_{j}}+A_{j, 1}(F) X_{j}^{N_{j}-1}+\ldots+A_{j, N_{j}}(F) \equiv 0
$$

(since $\left.\left[\mathbb{C}\left(X_{1}, \ldots, X_{n}\right): \mathbb{C}\left(F_{1}, \ldots, F_{n}\right)\right]<\infty\right)$. For such $j$, one can rewrite $(2.4)$ as

$$
\sum_{k=0}^{N_{j}} A_{j, k}(w) X_{j}^{N_{j}-k}=\sum_{k=1}^{n}\left(F_{k}-w_{k}\right) Q_{j, k}(F, w)
$$

where $Q_{j, k}, k=1, \ldots, n$, is in $\mathbb{C}\left[X_{1}, \ldots, X_{n}, Y_{1}, \ldots, Y_{n}\right]$. This can be done for each $j \in\{1, \ldots, n\}$. For any $w \in \mathbb{C}^{n} \backslash\left\{\prod_{j=1}^{n} A_{j, 0}(w)=0\right\}$, one has $\#\left\{F_{j}=w_{j}: j=1, \ldots, n\right\}<\infty$. For such $w$,

$$
\begin{aligned}
& {\left[\begin{array}{c}
G d X_{1} \wedge \ldots \wedge d X_{n} \\
F_{1}-w_{1}, \ldots, F_{n}-w_{n}
\end{array}\right]} \\
& =\left[\begin{array}{c}
G \operatorname{det}\left[Q_{j, k}\right]_{\substack{1 \leq j \leq n \\
1 \leq k \leq n}}(F, w) d X_{1} \wedge \ldots \wedge d X_{n} \\
\sum_{k=0}^{N_{1}} A_{1, k}(w) X_{1}^{N_{1}-k}, \ldots, \sum_{k=0}^{N_{n}} A_{n, k}(w) X_{n}^{N_{n}-k}
\end{array}\right]
\end{aligned}
$$

This follows from the transformation law for total sums of residues (see [Aiz]). On the other hand, for any $\left(\alpha_{1}, \ldots, \alpha_{n}\right) \in \mathbb{N}^{n}$,

$$
\begin{aligned}
& {\left[\begin{array}{c}
X_{1}^{\alpha_{1}} \cdot \ldots \cdot X_{n}^{\alpha_{n}} d X_{1} \wedge \ldots \wedge d X_{n} \\
\sum_{k=0}^{N_{1}} A_{1, k}(w) X_{1}^{N_{1}-k}, \ldots, \sum_{k=0}^{N_{n}} A_{n, k}(w) X_{n}^{N_{n}-k}
\end{array}\right]} \\
& \left.=\prod_{j=1}^{n}\left[\sum_{k=0}^{N_{j}} A_{j, k}^{\alpha_{j}} d X\right) X^{N_{j}-k}\right] .
\end{aligned}
$$

It is well known that the expression (2.7) is a rational function of $w$, with denominator

$$
\prod_{j=1}^{n}\left(A_{j, 0}(w)\right)^{1+\max \left(\alpha_{j}-N_{j}+1,0\right)} .
$$

This implies that (2.6) equals $R_{F, G}$, where $R_{F, G}$ is a rational function of $w$ 
with denominator

$$
\left(\prod_{j=1}^{n} A_{j, 0}(w)\right)^{n+\operatorname{deg} G} .
$$

This completes the proof of Lemma 2.1. Note that such a lemma was proved in [Bie1] using a completely different method.

When $F=\left(F_{1}, \ldots, F_{n}\right)$ is a proper map then, for any $G \in \mathbb{C}\left[X_{1}, \ldots, X_{n}\right]$, $R_{F, G}$ is a polynomial (since the $A_{j, 0}$ in (2.4) can be taken as equal to 1 ). We have in fact in this case the following more precise result:

Lemma 2.2. Let $F=\left(F_{1}, \ldots, F_{n}\right)$ be a polynomial proper map from $\mathbb{C}^{n}$ to $\mathbb{C}^{n}$ with Eojasiewicz exponent $\delta>0$. Then, for any $G \in \mathbb{C}\left[X_{1}, \ldots, X_{n}\right]$, the map

$$
w \mapsto\left[\begin{array}{c}
G d X_{1} \wedge \ldots \wedge d X_{n} \\
F_{1}-w_{1}, \ldots, F_{n}-w_{n}
\end{array}\right]
$$

is a polynomial map from $\mathbb{C}^{n}$ to $\mathbb{C}^{n}$ with degree at most:

$$
\mathrm{E}\left(\frac{1}{\delta}\left(\operatorname{deg} G+\max _{k}\left(\sum_{j \neq k} \operatorname{deg} F_{j}\right)+n\right)\right)-2 n+1 .
$$

Pro of. When $F=\left(F_{1}, \ldots, F_{n}\right)$ is a proper map, one has $\|F(\zeta)\| \geq \eta>0$ for $\|\zeta\| \geq R$. Therefore, it follows from the Bochner-Martinelli formula in its general form (see $[\mathrm{Aiz}]$ or $[\mathrm{BGVY}]$ ) that, for $\|w\| \ll 1$,

$$
\begin{aligned}
& \text { 2.8) } \quad\left[\begin{array}{c}
G d X_{1} \wedge \ldots \wedge d X_{n} \\
F_{1}-w_{1}, \ldots, F_{n}-w_{n}
\end{array}\right] \\
& =\frac{(-1)^{n(n-1) / 2}(n-1) !}{(2 i \pi)^{n}} \int_{\|\zeta\|=R} \frac{G(\zeta)\left(\sum_{j=1}^{n}(-1)^{j-1} \overline{F_{j}(\zeta)} \bigwedge_{k \neq j} \overline{d F_{k}(\zeta)}\right) \wedge d \zeta}{\left(\sum_{j=1}^{n} \overline{F_{j}(\zeta)}\left(F_{j}(\zeta)-w_{j}\right)\right)^{n}} \\
& =\sum_{m \in \mathbb{N}^{n}} \tau_{n, m}(R) \prod_{j=1}^{n} w_{j}^{m_{j}}
\end{aligned}
$$

where, for any $m=\left(m_{1}, \ldots, m_{n}\right) \in \mathbb{N}^{n},|m|=m_{1}+\ldots+m_{n}$ and

$$
\begin{aligned}
\tau_{n, m}(R)= & \frac{(-1)^{n(n-1) / 2}(n+|m|-1) !}{(2 i \pi)^{n}} \\
& \times \int_{\|\zeta\|=R} \frac{G \prod_{j=1}^{n} \bar{F}_{j}^{m_{j}}\left(\sum_{j=1}^{n}(-1)^{j-1} \bar{F}_{j} \bigwedge_{k \neq j} \overline{d F}_{k}\right) \wedge d \zeta}{\|F\|^{2(n+|m|)}} .
\end{aligned}
$$

Since $\|F(\zeta)\| \geq \gamma\|\zeta\|^{\delta}$ for $\|\zeta\| \gg 1$, the Stokes formula and standard estimates show that

$$
\int_{\|\zeta\|=R} \frac{G \prod_{j=1}^{n} \bar{F}_{j}^{m_{j}}\left(\sum_{j=1}^{m}(-1)^{j-1} \bar{F}_{j} \bigwedge_{k \neq j} \overline{d F}_{k}\right) \wedge d \zeta}{\|F\|^{2(n+|m|)}}=\lim _{R \rightarrow \infty} \int_{\|\zeta\|=R}(\cdot)=0
$$


if

$$
\delta(n+|m|)>\operatorname{deg} G+\max _{k}\left(\sum_{j \neq k} \operatorname{deg} F_{j}\right)-(n-1) \delta+n .
$$

This implies that (2.8) is a polynomial identity which is valid everywhere.

Remark. Note that this result (which already appeared in [BY1], [BY2] or [BGVY], Section 4) was also obtained by G. Biernat in [Bie1] using a different argument (in particular, the estimate for $\operatorname{deg} F_{G}$ we give here does not follow from such an argument). This was pointed out to us by M. Elkadi.

When $F=\left(F_{1}, \ldots, F_{n}\right)$ is a polynomial map proper at the origin (that is, $A_{0,1}, \ldots, A_{0, n}$ in $(2.4)$ can be taken such that $\left.A_{0,1}(0) \cdot \ldots \cdot A_{0, n}(0) \neq 0\right)$, one has:

Lemma 2.3. Let $F=\left(F_{1}, \ldots, F_{n}\right)$ be a proper map at the origin; then there exists an algebraic hypersurface $\Sigma$ in $\mathbb{C}^{n}$ with $0 \notin \Sigma$ such that for any $w \in \mathbb{C}^{n} \backslash \Sigma, \#\left\{\zeta: F_{j}(\zeta)=w_{j}, j=1, \ldots, n\right\}$ is finite and, for any $G \in \mathbb{C}\left[X_{1}, \ldots, X_{n}\right]$, there exists a rational function $R_{F, G}$ in $n$ variables with no poles at the origin such that, for $w \notin \Sigma$,

$$
\left[\begin{array}{c}
G d X_{1} \wedge \ldots \wedge d X_{n} \\
F_{1}-w_{1}, \ldots, F_{n}-w_{n}
\end{array}\right]=R_{F, G}(w) .
$$

P r o of. This follows from formulas (2.6) and (2.7) and from the fact that a denominator for $R_{F, G}$ in Lemma 2.1 is

$$
\left(\prod_{j=1}^{n} A_{j, 0}(w)\right)^{n+\operatorname{deg} G} .
$$

When $F$ is just dominant, few things can be said about the rational function $R_{F, G}$. Nevertheless, there are two interesting situations that will be discussed later.

DeFinition 2.1. Let $F=\left(F_{1}, \ldots, F_{n}\right)$ be a dominant polynomial map from $\mathbb{C}^{n}$ to $\mathbb{C}^{n}$. The map $F$ satisfies the separation condition over the origin if and only if there are constants $c>0,0<K_{1, j}<K_{2, j}<\infty, j=1, \ldots, n$, such that for any $j \in\{1, \ldots, n\}$,

$$
\|w\| \leq c \text { and } F(\zeta)=w \Rightarrow\left|\zeta_{j}\right| \leq K_{1, j} \text { or }\left|\zeta_{j}\right| \geq K_{2, j} .
$$

EXAmPle. Suppose the map $F=\left(F_{1}, \ldots, F_{n}\right)$ is commode in the sense of Płoski (see for example [PłT]), that is, there are $q, \kappa, K>0$ such that for any $j \in\{1, \ldots, n\}$, either $\left|\zeta_{j}\right| \leq K$ or $\|F(\zeta)\| \geq \kappa\left|\zeta_{j}\right|^{-q}$. Then the map $F$ satisfies the separation condition over the origin. This is immediate to check: if $F(\zeta)=w, w \in\left(\mathbb{C}^{n}\right)^{*}$, one has, for any $j \in\{1, \ldots, n\}$, either $\left|\zeta_{j}\right| \leq K$ or 
$\left|\zeta_{j}\right| \geq(\kappa /\|w\|)^{1 / q} ;$ for $w \in\left(\mathbb{C}^{n}\right)^{*},\|w\| \ll 1$, we have, for any $j \in\{1, \ldots, n\}$,

$$
F(\zeta)=w \Rightarrow\left|\zeta_{j}\right| \leq K \text { or }\left|\zeta_{j}\right| \geq K+1 .
$$

One can take $K$ large enough so that (2.10) remains valid when $w=0$. So that (2.9) is fulfilled with $K_{1, j}=K, j=1, \ldots, n, K_{2, j}=K+1, j=1, \ldots, n$.

Remark. Note that the separation condition over the origin implies that the fiber $F^{-1}(w)$ remains discrete when $\|w\| \ll 1$. This follows from the maximum principle: let $\Gamma$ be an irreducible branch with positive dimension of the fiber $F^{-1}(w)$, where $\|w\| \ll c$. Then, on $\Gamma$, the function

$$
\zeta \mapsto \frac{1}{\left(\left(K_{1, j}+K_{2, j}\right) / 2\right)-\zeta_{j}}
$$

is a bounded holomorphic function, therefore a constant, which is a contradiction (since this is true for any $j \in\{1, \ldots, n\}$ ).

Under such a separation condition, we have the following lemma.

LEMmA 2.4. Let $F=\left(F_{1}, \ldots, F_{n}\right)$ be a dominant polynomial map from $\mathbb{C}^{n}$ to $\mathbb{C}^{n}$ which satisfies the separation condition over the origin (with constants $\left.c, K_{1, j}, K_{2, j}, j=1, \ldots, n\right)$. Let $J$ be the Jacobian of $F$. There exists some algebraic hypersurface $\Sigma$ in $\mathbb{C}^{n}$ such that for any $w \notin \Sigma$, the variety $F^{-1}(w)$ is discrete, the polynomial $\prod_{j=1}^{n}\left(\left(\left(K_{1, j}+K_{2, j}\right) / 2\right)-X_{j}\right)$ does not vanish on it, and for any $G \in \mathbb{C}\left[X_{1}, \ldots, X_{n}\right]$, for any $w$ outside $\Sigma$,

$$
\left[\left(G J / \prod_{j=1}^{n}\left(\frac{K_{1, j}+K_{2, j}}{2}-X_{j}\right)^{\operatorname{deg} G}\right) d X_{1} \wedge \ldots \wedge d X_{n}\right]=\widetilde{R}_{F, G}(w),
$$

where $\widetilde{R}_{F, G}$ is a rational function with no poles at the origin.

Pr o of. Let

$$
d=\operatorname{deg}\left(F_{1}, \ldots, F_{n}\right)=\operatorname{dim}_{\mathbb{C}(w)}\left(\frac{\mathbb{C}(w)\left[X_{1}, \ldots, X_{n}\right]}{F_{1}-w_{1}, \ldots, F_{n}-w_{n}}\right) .
$$

If

$$
H(X):=\prod_{j=1}^{n}\left(\frac{K_{1, j}+K_{2, j}}{2}-X_{j}\right),
$$

then for $\|w\| \leq c, H$ does not vanish on the set $F^{-1}(w)$. For $w$ outside some algebraic hypersurface, the fiber $F^{-1}(w)$ is discrete and the polynomial $H$ does not vanish on it (this is just classical elimination theory). We can in fact be more precise: there are polynomials $\theta_{1}, \ldots, \theta_{d}$ in $w$, with $\theta_{d}(0) \neq 0$, such that, as linear operators in $\mathbb{C}(w)\left[X_{1}, \ldots, X_{n}\right] /\left(F_{1}-w_{1}, \ldots, F_{n}-w_{n}\right)$, 
one has:

$$
\bar{H}^{-1} \equiv \frac{1}{\theta_{d}(w)}\left(\sum_{k=0}^{d-1} \theta_{k}(w) \bar{H}^{d-1-k}\right) .
$$

For $w$ outside the hypersurface

$$
\Sigma=\left\{A_{1,0} \ldots A_{n, 0} \theta_{d}(w)=0\right\}
$$

the fiber $F^{-1}(w)$ is discrete and the polynomial $H$ does not vanish on it. Moreover, for such $w$,

$$
\left[\begin{array}{c}
\left(G J / H^{\operatorname{deg} G}\right) d X_{1} \wedge \ldots \wedge d X_{n} \\
F_{1}-w_{1}, \ldots, F_{n}-w_{n}
\end{array}\right]
$$

equals exactly the trace of the operator

$$
\frac{1}{\left(\theta_{d}(w)\right)^{\operatorname{deg} G}} \bar{G} \cdot\left(\sum_{k=0}^{d-1} \theta_{k}(w) \bar{H}^{d-1-k}\right)^{\operatorname{deg} G}
$$

as an operator in $\mathbb{C}\left[X_{1}, \ldots, X_{n}\right] /\left(F_{1}-w_{1}, \ldots, F_{n}-w_{n}\right)$; then from the computations in Lemma 2.1, it is a rational expression in $w$ with denominator

$$
\left(\theta_{d}(w)\right)^{\operatorname{deg} G}\left(\prod_{j=1}^{n} A_{j, 0}(w)\right)^{n(1+(d-1) \operatorname{deg} G)} .
$$

It remains to see that, in fact, this rational function has no poles at the origin. Let $w \in \mathbb{C}^{n} \backslash \Sigma,\|w\| \leq c$; then

$$
\left[\begin{array}{c}
\left(G J / H^{\operatorname{deg} G}\right) d X_{1} \wedge \ldots \wedge d X_{n} \\
F_{1}-w_{1}, \ldots, F_{n}-w_{n}
\end{array}\right]=\sum_{\alpha \in F^{-1}(w)} \frac{G(\alpha)}{H^{\operatorname{deg} G}(\alpha)} .
$$

It is immediate to see that, if $k_{1}, \ldots, k_{n}$ are positive integers with $k_{1}+\ldots+$ $k_{n} \leq \operatorname{deg} G$ and if $\alpha \in F^{-1}(w)$, then

$$
\begin{array}{r}
\frac{\left|\alpha_{1}^{N_{1}} \ldots \alpha_{n}^{N_{n}}\right|}{\mid\left(\left(\left(K_{1,1}+K_{2,1}\right) / 2\right)-\alpha_{1}\right)^{\operatorname{deg} G} \ldots\left(\left(\left(K_{1, n}+K_{2, n}\right) / 2\right)-\alpha_{n}\right)^{\operatorname{deg} G \mid}} \\
=\prod_{j=1}^{n} \frac{\left|\alpha_{j}\right|^{k_{j}}}{\left|\left(\left(K_{1, j}+K_{2, j}\right) / 2\right)-\alpha_{j}\right|^{\operatorname{deg} G} \leq C_{G}\left(k_{1}, \ldots, k_{n}\right)}
\end{array}
$$

for some constant $C_{G}\left(k_{1}, \ldots, k_{n}\right)$ independent of $\alpha$. This follows from the separation condition over the origin (in fact $\zeta \mapsto \zeta_{j} /\left(\left(\left(K_{1, j}+K_{2, j}\right) / 2\right)-\zeta_{j}\right.$ ) is bounded on $\bigcup_{\|w\| \leq c} F^{-1}(w)$, for any $\left.j \in\{1, \ldots, n\}\right)$. Therefore, since $\# F^{-1}(w) \leq d\left(F_{1}, \ldots, F_{n}\right)$ for $\|w\| \leq c$, one has, for $\|w\| \leq c, w \notin \Sigma$,

$$
\left|\left[\begin{array}{c}
\left(G J / H^{\operatorname{deg} G}\right) d X_{1} \wedge \ldots \wedge d X_{n} \\
F_{1}-w_{1}, \ldots, F_{n}-w_{n}
\end{array}\right]\right| \leq C
$$


for some constant $C$ independent of $w$. Then $\widetilde{R}_{F, G}$ has in fact no poles at the origin and the lemma is proved.

Remark 1. Suppose that $\left(F_{1}, \ldots, F_{n}\right)$ is separated over the origin and not proper over the origin. There are some constants $K, q, \kappa>0$ such that

$$
\|\zeta\| \geq K \Rightarrow\|F(\zeta)\| \geq \kappa\|\zeta\|^{-q} .
$$

This implies that if $\zeta \in F^{-1}(w), w \neq 0$, then either $\|\zeta\| \leq K$ or $\|\zeta\| \geq$ $(\kappa /\|w\|)^{1 / q}$. Following exactly the proof of Lemma 2.4, one can see then that

$$
\left[\begin{array}{c}
\frac{G J}{H^{\operatorname{deg} G+1}} d X_{1} \wedge \ldots \wedge d X_{n} \\
F_{1}-w_{1}, \ldots, F_{n}-w_{n}
\end{array}\right]=\sum_{\substack{\alpha \in F^{-1}(w) \\
\|\alpha\| \leq K}} \frac{G(\alpha)}{H^{\operatorname{deg} G+1}(\alpha)}+\varepsilon(w)
$$

where $\varepsilon(w) \rightarrow 0$ as $\|w\| \rightarrow 0$.

R e mark 2. In fact, what we really used in the proof of Lemma 2.4 or in Remark 1 above was the fact that for any $w,\|w\| \leq c$, and any $\zeta \in F^{-1}(w)$,

$$
|H(\zeta)| \geq \kappa\left(1+\left|\zeta_{1}\right|\right) \ldots\left(1+\left|\zeta_{n}\right|\right)
$$

for some positive constant $\kappa$.

The above remark leads us to introduce the following weaker separation condition:

Definition 2.2. Let $\delta>0$ and $H$ be a polynomial in $n$ variables. A dominant map $\left(F_{1}, \ldots, F_{n}\right)$ satisfies the $(H-\delta)$ separation condition over the origin if and only if there are positive constants $\kappa, c$ such that

$$
\left.\begin{array}{l}
\|w\| \leq c \\
F(\zeta)=w
\end{array}\right\} \Rightarrow|H(\zeta)| \geq \kappa(1+\|\zeta\|)^{\delta} .
$$

According to the above definition, we have the following:

LEMma 2.5. Let $F=\left(F_{1}, \ldots, F_{n}\right)$ be a dominant polynomial map from $\mathbb{C}^{n}$ to $\mathbb{C}^{n}$ which satisfies the $(H-\delta)$ separation condition over the origin. Let $J$ be the Jacobian of $F$. There exists some algebraic hypersurface $\Sigma$ in $\mathbb{C}^{n}$ such that for any $w \notin \Sigma$, the fiber $F^{-1}(w)$ is discrete, $H$ does not vanish on it, and for any $G \in \mathbb{C}\left[X_{1}, \ldots, X_{n}\right]$, and any $w$ outside $\Sigma$,

$$
\left[\begin{array}{c}
\frac{G J}{H^{\mathrm{E}(\operatorname{deg} G / \delta)+1}} d X_{1} \wedge \ldots \wedge d X_{n} \\
F_{1}-w_{1}, \ldots, F_{n}-w_{n}
\end{array}\right]=\widetilde{R}_{F, G}(w),
$$

where $\widetilde{R}_{F, G}$ is a rational function with no poles at the origin.

Pr o of. The proof is exactly the same as the proof of Lemma 2.4, except that estimate (2.12) has to be replaced by the following: if $\|w\| \leq c$ and 
$F(\alpha)=w$, then, for $k \in \mathbb{N}^{n}$,

$$
\frac{\left|\alpha_{1}^{k_{1}} \ldots \alpha_{n}^{k_{n}}\right|}{|H(\alpha)|^{\mathrm{E}(\operatorname{deg} G / \delta)+1}} \leq \widetilde{\kappa}_{G} \frac{\|\alpha\|^{\operatorname{deg} G}}{(1+\|\alpha\|)^{\delta(1+\mathrm{E}(\operatorname{deg} G / \delta))}} \leq C_{G}
$$

for some positive constants $\widetilde{\kappa}_{G}$ and $C_{G}$ since

$$
\delta(1+\mathrm{E}(\operatorname{deg} G / \delta)) \geq \operatorname{deg} G .
$$

So the function $\zeta \rightarrow G(\zeta) / H(\zeta)^{\mathrm{E}(\operatorname{deg} G / \delta)+1}$ is bounded on $\bigcup_{\|w\| \leq c} F^{-1}(w)$, and the lemma follows exactly as in the proof of Lemma 2.4. There is also in this case a remark analogous to Remark 1 above.

3. A first approach to the Nullstellensatz. The first result we will prove in this section is some explicit version of the algebraic Nullstellensatz for proper polynomial maps $F: \mathbb{C}^{n} \rightarrow \mathbb{C}^{n}$ with Łojasiewicz exponent $\delta>0$. The methods here are inspired by those used to provide a solution with economic bounds (taking into account the projective degree, that is, in general, the product of the degrees of the entries) for the algebraic Bézout identity (see for example [BY2], [BGVY], Section 5, [ElY], [Y]).

Theorem 3.1. Let $F=\left(F_{1}, \ldots, F_{n}\right)$ be a polynomial map from $\mathbb{C}^{n}$ to $\mathbb{C}^{n}$ such that

$$
\|Z\| \geq K \Rightarrow\|P(Z)\| \geq \kappa\|Z\|^{\delta}
$$

for some $K \geq 0, \kappa>0, \delta>0$. Let $G$ be some element in $\mathbb{C}\left[X_{1}, \ldots, X_{n}\right]$ such that $G \mid F^{-1}(0) \equiv 0$. Then, if

$$
D:=\max _{1 \leq j \leq n}\left(\operatorname{deg} F_{j}\right) \quad \text { and } \quad \nu:=\max _{\alpha \in F^{-1}(0)} \nu_{\alpha}(F),
$$

we have

$$
\left\{\begin{array}{l}
G^{\nu}=\sum_{j=1}^{n} A_{j} F_{j} \\
\operatorname{deg} A_{j} F_{j} \leq D\left(\mathrm{E}\left(\frac{2}{\delta}\left(\sum_{j=1}^{n} \operatorname{deg} F_{j}+\nu \operatorname{deg} G\right)\right)-n\right) .
\end{array}\right.
$$

Proof. Let $Q_{1,1}, \ldots, Q_{n, n}$ be polynomials in $2 n$ variables such that $\operatorname{deg}\left(Q_{j, k}\right) \leq \operatorname{deg} F_{j}-1$ for $j, k=1, \ldots, n$ and

$$
\forall Z \in \mathbb{C}^{n}, \forall \zeta \in \mathbb{C}^{n}, \quad F_{j}(Z)-F_{j}(\zeta)=\sum_{k=1}^{n} Q_{j, k}(Z, \zeta)\left(z_{k}-\zeta_{k}\right) ;
$$

let $\Delta(Z, \zeta)$ be the determinant of the matrix $\left[Q_{j, k}\right]_{1 \leq j, k \leq n}$ (a Bézoutian for the map $\left.\left(F_{1}, \ldots, F_{n}\right)\right)$. For any $Z \in \mathbb{C}^{n}$, the set $F^{-1}(F(Z))$ is finite (since $F$ is a proper map). Then one can apply the global transformation law for 
multidimensional residues $[$ Aiz $]$ and get, for any $G$ such that $G \mid F^{-1}(0) \equiv 0$, and any $Z$ in $\mathbb{C}^{n}$,

$$
G^{\nu}(Z)=\left[\begin{array}{c}
G(X)^{\nu} \Delta(Z, X) d X_{1} \wedge \ldots \wedge d X_{n} \\
F_{1}-F_{1}(Z), \ldots, F_{n}-F_{n}(Z)
\end{array}\right]
$$

Let

$$
\Delta(Z, X)=\sum_{|\alpha|+|\beta| \leq \sum_{j=1}^{n} \operatorname{deg} F_{j}-n} c_{\alpha, \beta} z_{1}^{\alpha_{1}} \ldots z_{n}^{\alpha_{n}} X_{1}^{\beta_{1}} \ldots X_{n}^{\beta_{n}} .
$$

One has, from (3.3), for any $Z \in \mathbb{C}^{n}$,

$$
G^{\nu}(Z)=\sum_{|\alpha|+|\beta| \leq \sum_{j=1}^{n} \operatorname{deg} F_{j}-n} c_{\alpha, \beta}\left[\begin{array}{c}
G(X)^{\nu} X^{\beta} d X_{1} \wedge \ldots \wedge d X_{n} \\
F_{1}-F_{1}(X), \ldots, F_{n}-F_{n}(X)
\end{array}\right]
$$

where we used the abridged notation $X^{\beta}:=X_{1}^{\beta_{1}} \ldots X_{n}^{\beta_{n}}$. From Lemma 2.2, we know that

$$
\left[\begin{array}{c}
G(X)^{\nu} X^{\beta} d X_{1} \wedge \ldots \wedge d X_{n} \\
F_{1}-w_{1}, \ldots, F_{n}-w_{n}
\end{array}\right]=\mathcal{Q}_{\beta}[G](w)
$$

where $\mathcal{Q}_{\beta}[G]$ is a polynomial with total degree at most

$$
\mathrm{E}\left(\frac{1}{\delta}\left(\nu \operatorname{deg} G+|\beta|+\max _{k}\left(\sum_{j \neq k} \operatorname{deg} F_{j}\right)+n\right)\right)-2 n+1 .
$$

Since $G^{\nu}$ is in the ideal $\left(F_{1}, \ldots, F_{n}\right)$ because of Noether's theorem, one has $\mathcal{Q}_{\beta}[G](0)=0$. Therefore

$$
\mathcal{Q}_{\beta}[G](w)=\sum_{j=1}^{n} w_{j} \mathcal{Q}_{\beta}^{[j]}[G](w)
$$

for some polynomials $\mathcal{Q}_{\beta}^{[j]}$. Formula (3.4) can be written as

$$
G^{\nu}(Z)=\sum_{|\alpha|+|\beta| \leq \sum_{j=1}^{n} \operatorname{deg} F_{j}-n} \sum_{k=1}^{n} c_{\alpha, \beta} F_{k}(Z) Z^{\alpha} \mathcal{Q}_{\beta}^{[k]}[G](F(Z)) .
$$

It is clear that (3.5) is a formula of the form $G^{\nu}=\sum_{j=1}^{n} A_{j} F_{j}$ where $\operatorname{deg} A_{j} F_{j}$

$$
\begin{aligned}
& \leq \max _{|\alpha|+|\beta| \leq \sum_{j=1}^{n} \operatorname{deg} F_{j}-n}\left[|\alpha|+D \operatorname{deg}\left(\mathcal{Q}_{\beta}[G]\right)\right] \\
& \leq \max _{\alpha, \beta}\left(|\alpha|+D\left(\mathrm{E}\left(\frac{1}{\delta}\left(\nu \operatorname{deg} G+|\beta|+\max _{k} \sum_{j \neq k} \operatorname{deg} F_{j}+n\right)\right)-2 n+1\right)\right) \\
& \leq D\left(\mathrm{E}\left(\frac{2}{\delta}\left(\sum_{j=1}^{n} \operatorname{deg} F_{j}+\nu \operatorname{deg} G\right)\right)-n\right) .
\end{aligned}
$$


Remark. When $F=\left(F_{1}, \ldots, F_{n}\right)$ is proper over the origin, and $G \mid F^{-1}(0) \equiv 0$, then one knows from Lemma 2.3 that

$$
\mathcal{Q}_{\beta}[G](w)=\frac{\mathcal{N}_{\beta}[G](w)}{\left(\prod_{j=1}^{n} A_{j, 0}(w)\right)^{n+|\beta|+\nu \operatorname{deg} G}}
$$

where $\mathcal{N}_{\beta}[G]$ is a polynomial in $w$ vanishing at 0 and the $A_{j, 0}$ come from (2.4). The same method as above can be applied for $\|w\| \ll 1$ and we obtain for $\|Z\|$ close to 0 ,

$$
\begin{aligned}
& G^{\nu}(Z) \\
& =\sum_{|\alpha|+|\beta| \leq \sum_{j=1}^{n} \operatorname{deg} F_{j}-n} c_{\alpha, \beta} F_{k}(Z) Z^{\alpha} \frac{\mathcal{N}_{\beta}^{[k]}[G](F(Z))}{\left(\prod_{j=1}^{n} A_{j, 0} F(Z)\right)^{n+|\beta|+\nu \operatorname{deg} G}}
\end{aligned}
$$

where

$$
\mathcal{N}_{\beta}[G](w)=\sum_{j=1}^{n} w_{j} \mathcal{N}_{\beta}^{[j]}[G](w) .
$$

Raising denominators in (3.6) provides also some effective Nullstellensatz since $A_{j, 0}(0) \neq 0$. The problem one has doing that is that the bounds for the degrees of the $A_{j, 0}$ and the $\mathcal{N}_{\beta}^{[j]}[G]$ can only be estimated using for example Perron's theorem ([Pe], Satz 57) and the transformation law.

In fact, with respect to the Nullstellensatz, nice estimates (for the case of maps proper over the origin) can be obtained following a method due to A. Płoski and P. Cassou-Noguès. In order to be complete, we will recall here their result and its proof.

TheOREM $3.2[\mathrm{CNPl}]$. Let $F=\left(F_{1}, \ldots, F_{n}\right)$ be a dominant polynomial map from $\mathbb{C}^{n}$ to $\mathbb{C}^{n}$ proper over the origin, and $G \in \mathbb{C}[X]$ such that $G \mid F^{-1}(0) \equiv 0$. Then for some exponent $s \leq d=\operatorname{deg} F$,

$$
G^{s}=\sum_{j=1}^{n} A_{j} F_{j}, \quad \operatorname{deg} A_{j} F_{j} \leq \operatorname{deg} G \operatorname{deg} F_{1} \ldots \operatorname{deg} F_{n} .
$$

Proof. For any $w$ close to 0 ,

$$
\# F^{-1}(w)=d=\left[\mathbb{C}\left(X_{1}, \ldots, X_{n}\right): \mathbb{C}\left(F_{1}, \ldots, F_{n}\right)\right]
$$

if the points are counted with multiplicities. Let $S_{1}(w), \ldots, S_{d}(w)$ be the Newton symmetric polynomials in the values $\left(G\left(\zeta_{1}(w)\right), \ldots, G\left(\zeta_{d}(w)\right)\right)$, where $\left\{\zeta_{1}(w), \ldots, \zeta_{d}(w)\right\}=F^{-1}(w)$. Since for $1 \leq j \leq d$,

$$
S_{j}(w)=\left[\begin{array}{c}
G^{j} J d X_{1} \wedge \ldots \wedge d X_{n} \\
F_{1}-w_{1}, \ldots, F_{n}-w_{n}
\end{array}\right]
$$

( $J$ as usual being the jacobian of $F$ ), it follows from Lemma 2.3 that $S_{j}$ is a rational function with no poles at 0 . The same is true for the symmetric 
functions $\sigma_{1}(w), \ldots, \sigma_{d}(w)$ of $\left\{\zeta_{1}(w), \ldots, \zeta_{d}(w)\right\}$. For any $w$ close to zero and such that $F^{-1}(w)$ contains only simple points,

$$
G^{d}-\sigma_{1}(w) G^{d-1}+\ldots+(-1)^{d} \sigma_{d}(w) \in\left(F_{1}-w_{1}, \ldots, F_{n}-w_{n}\right) .
$$

If

$$
\sigma_{j}(w)=A_{j}(w) / A_{0}(w)
$$

with $A_{j} \in \mathbb{C}[w]$, then one has the polynomial identity

$$
A_{0}(F) G^{d}-A_{1}(F) G^{d-1}+\ldots+(-1)^{d} A_{d}(F) \equiv 0
$$

where $A_{0}(0) \neq 0, A_{1}(0)=\ldots=A_{d}(0)=0$ (all $G\left(\zeta_{j}(0)\right)$ are zero since $\left.G \mid F^{-1}(0) \equiv 0\right)$. One has also an equation for $G$ :

$$
\widetilde{A}_{0}(F) G^{s}+\widetilde{A}_{1}(F) G^{s-1}+\ldots+\widetilde{A}_{s}(F) \equiv 0
$$

$(s \leq d)$, which is irreducible over $\mathbb{C}\left[F_{1}, \ldots, F_{n}\right]$ and such that $\widetilde{A}_{0}(0) \neq 0$, $\widetilde{A}_{1}(0)=\ldots=\widetilde{A}_{s}(0)=0$. Let

$$
\mathcal{Q}\left(X_{1}, \ldots, X_{n}, Y\right)=\widetilde{A}_{0}(X) Y^{s}+\ldots+\widetilde{A}_{s}(X) .
$$

Since the polynomials $F_{1}, \ldots, F_{n}, G$ are algebraically dependent, there exists from Perron's theorem ([Pe], Satz 56\&57, pp. 125-126) some polynomial $\widetilde{\mathcal{Q}}$ in $n+1$ variables such that

$$
\left\{\begin{array}{l}
\widetilde{\mathcal{Q}}\left(F_{1}, \ldots, F_{n}, G\right) \equiv 0, \\
\operatorname{deg}_{w_{n+1}} \widetilde{\mathcal{Q}}>0, \\
\operatorname{deg}_{w} \widetilde{\mathcal{Q}}\left(w_{1}^{\operatorname{deg} F_{1}}, \ldots, w_{n}^{\operatorname{deg} F_{n}}, w_{n+1}^{\operatorname{deg} G}\right) \leq \operatorname{deg} G \operatorname{deg} F_{1} \ldots \operatorname{deg} F_{n} .
\end{array}\right.
$$

We now use an argument of Jelonek (see the proof of Proposition 12 in [Je]). Since $\mathcal{Q}$ is irreducible, $\mathcal{Q}$ divides $\widetilde{\mathcal{Q}}$, so that we can compare the weighted degrees:

$\operatorname{deg}_{w} \mathcal{Q}\left(w_{1}^{\operatorname{deg} F_{1}}, \ldots, w_{n}^{\operatorname{deg} F_{n}}, w_{n+1}^{\operatorname{deg} G}\right) \leq \operatorname{deg}_{w} \widetilde{\mathcal{Q}}\left(w_{1}^{\operatorname{deg} F_{1}}, \ldots, w_{n}^{\operatorname{deg} F_{n}}, w_{n+1}^{\operatorname{deg} G}\right)$.

Now, one can rewrite (3.8) as

$$
\widetilde{A}_{0}(0) G^{s}=-G^{s}\left(\sum_{k=1}^{n} F_{k} \widetilde{B}_{0, k}(F)\right)+\sum_{l=1}^{s} G^{s-l}\left(\sum_{k=1}^{n} F_{k} \widetilde{B}_{j, k}(F)\right)
$$

where

$$
\widetilde{A}_{j}(w)=\widetilde{A}_{j}(0)+\sum_{k=1}^{n} w_{k} \widetilde{B}_{j, k}(w) .
$$

This provides the Nullstellensatz with the convenient estimates.

When $\left(F_{1}, \ldots, F_{n}\right)$ is dominant and separated over the origin (Definition 2.1) one has:

TheOREM 3.3. Let $F=\left(F_{1}, \ldots, F_{n}\right)$ be a dominant polynomial map from $\mathbb{C}^{n}$ to $\mathbb{C}^{n}$ which is separated over the origin; let $G \in \mathbb{C}[X], G \mid F^{-1}(0) \equiv 0$. 
Then, for some $s \leq d=\left[\mathbb{C}\left(X_{1}, \ldots, X_{n}\right): \mathbb{C}\left(F_{1}, \ldots, F_{n}\right)\right]$,

$$
G^{s}=\sum_{j=1}^{n} A_{j} F_{j}, \quad \operatorname{deg} A_{j} F_{j} \leq n(\operatorname{deg} G+1)\left[\operatorname{deg} F_{1} \ldots \operatorname{deg} F_{n}+d\right] .
$$

Pro of. One can suppose that $F$ is not proper over the origin. Let $G \in$ $\mathbb{C}\left[X_{1}, \ldots, X_{n}\right], G \mid F^{-1}(0) \equiv 0$. Let $K_{1, j}, K_{2, j}, j=1, \ldots, n$, be the constants corresponding to the fact that the map $F$ is separated at the origin (see Definition 2.1) and

$$
H(X):=\prod_{j=1}^{n}\left(\frac{K_{j, 1}+K_{j, 2}}{2}-X_{j}\right) .
$$

Consider, for $\|w\| \ll 1$ such that $\# F^{-1}(w)=d$, the Newton sums $S_{1}^{(G)}, \ldots$ $\ldots, S_{d}^{(G)}$ of the values

$$
\left\{\frac{G\left(\zeta_{1}(w)\right)}{H^{\operatorname{deg} G+1}\left(\zeta_{1}(w)\right)}, \ldots, \frac{G\left(\zeta_{d}(w)\right)}{H^{\operatorname{deg} G+1}\left(\zeta_{d}(w)\right)}\right\}
$$

where $F^{-1}(w)=\left\{\zeta_{1}(w), \ldots, \zeta_{d}(w)\right\}$. By Remark 1 after the proof of Lemma 2.4, the functions $S_{j}^{G}$ can be written near the origin as

$$
S_{j}^{(G)}(w)=\sum_{\substack{\alpha \in F^{-1}(w) \\\|\alpha\| \leq K}}\left(\frac{G(\alpha)}{H^{\operatorname{deg} G+1}(\alpha)}\right)^{j}+\varepsilon_{j}(w)
$$

where $K$ is a constant and $\lim _{w \mapsto 0} \varepsilon_{j}(w)=0$. Therefore, since $G \mid F^{-1}(0) \equiv 0$, one has

$$
S_{j}^{(G)}(0)=0, \quad j=1, \ldots, d .
$$

The same is true for the symmetric polynomials $\sigma_{1}^{(G)}, \ldots, \sigma_{d}^{(G)}$ of the values

$$
\left\{\frac{G\left(\zeta_{1}(w)\right)}{H^{\operatorname{deg} G+1}\left(\zeta_{1}(w)\right)}, \ldots, \frac{G\left(\zeta_{d}(w)\right)}{H^{\operatorname{deg} G+1}\left(\zeta_{d}(w)\right)}\right\}
$$

where $F^{-1}(w)=\left\{\zeta_{1}(w), \ldots, \zeta_{d}(w)\right\}$. As in the proof of Theorem 3.2, for any $w$ close to zero such that $\# F^{-1}(w)=d$ and $F^{-1}(w)$ contains only simple points, one has

$$
\begin{aligned}
\left(\frac{G}{H^{\operatorname{deg} G+1}}\right)^{d}-\sigma_{1}^{(G)}(w)\left(\frac{G}{H^{\operatorname{deg} G+1}}\right)^{d-1} & +\ldots+\sigma_{d}^{(G)}(w) \\
& \in\left(F_{1}-w_{1}, \ldots, F_{n}-w_{n}\right) .
\end{aligned}
$$

If $\widetilde{G}$ is the rational function $G / H^{\operatorname{deg} G+1}$, then one has the identity

$$
A_{0}^{(G)}(F) \widetilde{G}^{d}-A_{1}^{(G)}(F) \widetilde{G}^{d-1}+\ldots+(-1)^{d} A_{d}^{(G)}(F) \equiv 0,
$$


where

$$
\sigma_{j}^{(G)}(w)=\frac{A_{j}^{(G)}(w)}{A_{0}^{(G)}(w)}
$$

with $A_{0}^{(G)}(0) \neq 0, A_{1}^{(G)}(0)=\ldots=A_{d}^{(G)}(0)=0$. There is some equation for $\widetilde{G}$ of the form

$$
\widetilde{A}_{0}^{(G)}(F) \widetilde{G}^{s}+\widetilde{A}_{1}^{(G)}(F) \widetilde{G}^{s-1}+\ldots+\widetilde{A}_{s}^{(G)}(F)=0
$$

which is irreducible over $\mathbb{C}\left[F_{1}, \ldots, F_{n}\right]$ and such that $\widetilde{A}_{0}^{(G)}(0) \neq 0, \widetilde{A}_{1}^{(G)}(0)=$ $\ldots=\widetilde{A}_{d}^{(G)}(0)=0$. Since $\widetilde{G}$ is now a rational function $\widetilde{G}=G / H^{\operatorname{deg} G+1}$, we cannot apply Perron's theorem immediately as in the proof of Theorem 3.2. In order to find a substitute argument, we use some variant of the famous Rabinowicz trick. Let us add some additional variable $X_{0}$; the polynomials

$$
\left(F_{1}(X), \ldots, F_{n}(X), H^{\operatorname{deg} G+1}(X)-X_{0} G(X), X_{0}\right)
$$

are algebraically dependent; there exists, by Perron's theorem ([Pe], Satz 56 and 57), some polynomial $\mathcal{Q}^{G}$ in $n+2$ variables such that

$$
\left\{\begin{array}{l}
\mathcal{Q}^{G}\left(F_{1}, \ldots, F_{n}, X_{0}, H^{\operatorname{deg} G+1}-X_{0} G\right) \equiv 0 \\
\operatorname{deg}_{w} \mathcal{Q}^{G}\left(w_{1}^{\operatorname{deg} F_{1}}, \ldots, w_{n}^{\operatorname{deg} F_{n}}, w_{n+1}, w_{n+2}^{n(\operatorname{deg} G+1)}\right) \\
\quad \leq n \operatorname{deg} F_{1} \ldots \operatorname{deg} F_{n}(\operatorname{deg} G+1) .
\end{array}\right.
$$

Then, substituting $X_{0}=H^{\operatorname{deg} G+1} / G$ in (3.10), one gets

$$
\mathcal{Q}_{0}^{G}\left(F_{1}, \ldots, F_{n}, 1 / \widetilde{G}\right) \equiv 0
$$

where

One has

$$
\mathcal{Q}_{0}^{G}\left(X_{1}, \ldots, X_{n}, X_{n+1}\right):=\mathcal{Q}^{G}\left(X_{1}, \ldots, X_{n}, X_{n+1}, 0\right)
$$

$$
\begin{aligned}
\operatorname{deg}_{w} \mathcal{Q}^{G}\left(w_{1}^{\operatorname{deg} F_{1}}, \ldots, w_{n}^{\operatorname{deg} F_{n}},\right. & \left.w_{n+1}\right) \\
& \leq n \operatorname{deg} F_{1} \ldots \operatorname{deg} F_{n}(\operatorname{deg} G+1) .
\end{aligned}
$$

Since $\left(F_{1}, \ldots, F_{n}\right)$ is dominant, the relation (3.11) multiplied by $\widetilde{G}^{\operatorname{deg}_{w_{n+1}}} \mathcal{Q}_{0}^{G}$ provides an equation for $\widetilde{G}$ (which is such that (3.9) divides it, so that we have estimates on the weighted degrees of the $\widetilde{A}_{k}^{(G)}$ thanks to estimates (3.10)). The relation (3.9) can be rewritten as

$$
\sum_{k=0}^{s} \widetilde{A}_{k}^{(G)}(F) G^{s-k} H^{k(\operatorname{deg} G+1)} \equiv 0 .
$$

Since $\widetilde{A}_{0}^{(G)}(0) H(0) \neq 0$, the equation (3.13) solves the Nullstellensatz. One can rewrite (3.13) as 


$$
\begin{aligned}
G^{s} & =\sum_{j=1}^{n} A_{j} F_{j}, \\
\operatorname{deg}_{j} F_{j} & \leq n(\operatorname{deg} G+1) \operatorname{deg} F_{1} \ldots \operatorname{deg} F_{n}+\operatorname{sn}(\operatorname{deg} G+1) \\
& \leq n(\operatorname{deg} G+1)\left[\operatorname{deg} F_{1} \ldots \operatorname{deg} F_{n}+d\right] .
\end{aligned}
$$

This concludes the proof of Theorem 3.3.

When $F=\left(F_{1}, \ldots, F_{n}\right)$ is dominant and satisfies the $(H-\delta)$ separation condition in Definition 2.2, one has a similar result.

THEOREM 3.4. Let $F=\left(F_{1}, \ldots, F_{n}\right)$ be a dominant polynomial map from $\mathbb{C}^{n}$ to $\mathbb{C}^{n}$ which satisfies the $(H-\delta)$ condition for some $H \in \mathbb{C}[X]$ and $\delta>0$. Then, for $G \in \mathbb{C}[X]$ such that $G \mid F^{-1}(0) \equiv 0$, one has for some $s \leq d\left(F_{1}, \ldots, F_{n}\right)$,

$$
G^{s}=\sum_{j=1}^{n} A_{j} F_{j}
$$

$\operatorname{deg} A_{j} F_{j} \leq \operatorname{deg} H(\mathrm{E}(\operatorname{deg} G / \delta)+1)\left(\operatorname{deg} F_{1} \ldots \operatorname{deg} F_{n}+d\right)$.

Proof. Let $G \in \mathbb{C}[X]$ be such that $G \mid F^{-1}(0) \equiv 0$. We now let

$$
\widetilde{G}=G / H^{\mathrm{E}(\operatorname{deg} G / \delta)+1} .
$$

We use Lemma 2.5 instead of Lemma 2.4. We obtain as before a relation of the form (3.9), with the same conditions on $\widetilde{A}_{0}^{(G)}, \ldots, \widetilde{A}_{s}^{(G)}$. On the other hand, by the same argument as in the proof of Theorem 3.3, there is a relation

$$
\mathcal{Q}^{(G)}\left(F_{1}, \ldots, F_{n}, \widetilde{G}\right) \equiv 0
$$

with

$$
\begin{aligned}
\operatorname{deg}_{w} \mathcal{Q}^{(G)}\left(w_{1}^{\operatorname{deg} F_{1}}, \ldots, w_{n}^{\operatorname{deg} F_{n}}, w_{n+1}\right) & \\
& \leq \operatorname{deg} F_{1} \ldots \operatorname{deg} F_{n} \operatorname{deg} H(\mathrm{E}(\operatorname{deg} G / \delta)+1) .
\end{aligned}
$$

Since (3.9) divides (3.14), one has

$$
G^{s}=\sum_{j=1}^{n} A_{j} F_{j},
$$

$\operatorname{deg} A_{j} F_{j} \leq \operatorname{deg} F_{1} \ldots \operatorname{deg} F_{n} \operatorname{deg} H(\mathrm{E}(\operatorname{deg} G / \delta)+1)$

$$
+s(\mathrm{E}(\operatorname{deg} G / \delta)+1) \operatorname{deg} H
$$$$
\leq \operatorname{deg} H(\mathrm{E}(\operatorname{deg} G / \delta)+1)\left(\operatorname{deg} F_{1} \ldots \operatorname{deg} F_{n}+d\right) .
$$

4. Some consequences of the Briançon-Skoda theorem. In this section, we prove that for maps with a negative Łojasiewicz exponent, the Nullstellensatz can be solved with estimates (1.11) or (1.12). 
Theorem 4.1. Let $F=\left(F_{1}, \ldots, F_{k}\right)$ be a polynomial map from $\mathbb{C}^{n}$ to $\mathbb{C}^{k}$ such that

$$
\|X\|>K \Rightarrow\|F(X)\| \geq \kappa\|X\|^{q}
$$

for some $K \geq 0, q \leq 0$ and $\kappa>0$. Let $G \in \mathbb{C}\left[X_{1}, \ldots, X_{n}\right], G \equiv 0$ on $F^{-1}(0)$. Then, if

$$
\gamma:=\min (n+1, k), \quad \nu:=\max _{\alpha \in F^{-1}(0)}\left(\nu_{\alpha}\right), \quad D:=\max _{1 \leq j \leq k} \operatorname{deg} F_{j},
$$

we have

$$
G^{\gamma \nu}=\sum_{j=1}^{k} A_{j} F_{j}, \quad \operatorname{deg} A_{j} F_{j} \leq \gamma(\nu \operatorname{deg} G+D+|q|) .
$$

Proof. From (4.1), for any $\widetilde{X}=\left(X_{0}, X\right)=\left(X_{0}, \ldots, X_{n}\right) \in \mathbb{C}^{n+1}, X_{0} \neq$ $0,\|X\| \geq K\left|X_{0}\right|$,

$$
\left\|F\left(X / X_{0}\right)\right\| \geq \kappa\left(\|X\| /\left|X_{0}\right|\right)^{q}
$$

that is,

$$
\left|X_{0}\right|^{|q|} \leq \frac{1}{\kappa}\|X\|^{|q|}\left\|F\left(X / X_{0}\right)\right\|,
$$

SO

$$
\left|X_{0}\right|^{|q|+D} \leq \frac{1}{\kappa}\|X\|^{|q|}\left(\sum_{j=1}^{k}\left|{ }^{\mathrm{h}} F_{j}(\widetilde{X})\right|^{2}\left|X_{0}\right|^{2\left(D-\operatorname{deg} F_{j}\right)}\right)^{1 / 2} .
$$

Let $G \in \mathbb{C}\left[X_{1}, \ldots, X_{n}\right], G \equiv 0$ on $F^{-1}(0)$; then $G^{\nu} \in\left(F_{1}, \ldots, F_{k}\right)$ by Noether's theorem. There exists some constant $C>0$ such that, for any $\widetilde{X} \in S^{2 n+1}:=\left\{\widetilde{X} \in \mathbb{C}^{n+1}:\|\widetilde{X}\|=1\right\}$ with $\left|X_{0}\right| \geq 1 /(2 K)$,

$$
\left|{ }^{\mathrm{h}} G^{\nu}(\widetilde{X})\right| \leq C\left\|{ }^{\mathrm{h}} F(\widetilde{X})\right\| \text {. }
$$

On the other hand, because of (4.4), there exists $C^{\prime}>0$ such that for any $\widetilde{X} \in S^{2 n+1}$ with $\left|X_{0}\right| \leq 1 / K$,

$$
\left|X_{0}\right|^{|q|+D} \leq C^{\prime}\left\|^{\mathrm{h}} F(\tilde{X})\right\| .
$$

On the unit sphere $S^{2 n+1}$,

$$
\left.\left|{ }^{\mathrm{h}} G^{\nu}(\widetilde{X})\right| X_{0}\right|^{|q|+D}\left|\leq C^{\prime \prime}\right|{ }^{\mathrm{h}} F(\widetilde{X}) \|
$$

for some $C^{\prime \prime}>0$. So for any $\widetilde{X} \in \mathbb{C}^{n+1} \backslash\{0\}$,

$$
\left|{ }^{\mathrm{h}} G^{\nu}(\widetilde{X} /\|\widetilde{X}\|)\left(X_{0} /\|\widetilde{X}\|\right)^{|q|+D}\right| \leq C^{\prime \prime}\left\|{ }^{\mathrm{h}} F(\widetilde{X} /\|\widetilde{X}\|)\right\|,
$$

hence

$$
\left|{ }^{\mathrm{h}} G^{\nu}(\tilde{X})\right| \cdot\left|X_{0}\right|^{|q|+D} \leq C^{\prime \prime}\left|X_{0}\right|^{|q|+D+\nu \operatorname{deg} G}\left\|^{\mathrm{h}} F(\tilde{X} /\|\widetilde{X}\|)\right\|,
$$


and consequently,

$$
\begin{aligned}
\left.\right|^{\mathrm{h}} G^{\nu}(\widetilde{X}) \mid \cdot & \left|X_{0}\right|^{|q|+D} \\
& \leq C^{\prime \prime}\left|X_{0}\right|^{|q|+\nu \operatorname{deg} G}\left(\sum_{j=1}^{k}\left|{ }^{\mathrm{h}} F_{j}(\widetilde{X})\right|^{2}\left|X_{0}\right|^{2\left(D-\operatorname{deg} F_{j}\right)}\right)^{1 / 2} .
\end{aligned}
$$

The inequality (4.6) remains valid at zero. At any point $\widetilde{Z}$ in $\mathbb{C}^{n+1}$, the germ at $\widetilde{Z}$ of ${ }^{\mathrm{h}} G^{\nu} X_{0}^{|q|+D}$ is in the integral closure of the ideal generated by the germs of ${ }^{\mathrm{h}} F_{1}, \ldots,{ }^{\mathrm{h}} F_{k}$ at this point. Then, by the Briançon-Skoda theorem ([BS], or $[\mathrm{LT}]$ for an algebraic version)

$$
\left({ }^{\mathrm{h}} G^{\nu} X_{0}^{|q|+D}\right)^{\gamma} \in\left({ }^{\mathrm{h}} F_{1}, \ldots,{ }^{\mathrm{h}} F_{k}\right)_{\mathrm{loc}} .
$$

So, there are polynomials $\widetilde{A}_{1}, \ldots, \widetilde{A}_{k} \in \mathbb{C}\left[X_{0}, \ldots, X_{n}\right]$ such that

$$
\left({ }^{\mathrm{h}} G^{\nu} X_{0}^{|q|+D}\right)^{\gamma}=\sum_{j=1}^{k} \widetilde{A}_{j}(\tilde{X})^{\mathrm{h}} F_{j}(\tilde{X}) .
$$

Taking $X_{0}=1$ in (4.6), we get the result.

Under some additional hypothesis, we have

TheOREM 4.2. Let $F=\left(F_{1}, \ldots, F_{k}\right)$ be a polynomial map from $\mathbb{C}^{n}$ to $\mathbb{C}^{k}$ such that

$$
\|X\| \geq K \Rightarrow\|F(X)\| \geq \kappa\|X\|^{q}
$$

for some constants $K \geq 0, q \leq 0, \kappa>0$. Assume that the depth of the ideal

$$
{ }^{\mathrm{h}} F_{1 n+1} \mathcal{O}_{0}+\ldots+{ }^{\mathrm{h}} F_{k n+1} \mathcal{O}_{0}
$$

in $\mathcal{O}_{0}$ is greater than 2. Let $G \in \mathbb{C}\left[X_{1}, \ldots, X_{n}\right], G \equiv 0$ on $F^{-1}(0)$. Then, if $\nu:=\max _{\alpha \in F^{-1}(0)}\left(\nu_{\alpha}\right), D:=\max _{1 \leq j \leq k} \operatorname{deg}\left(F_{j}\right)$, we have

$$
G^{\nu}=\sum_{j=1}^{k} A_{j} F_{j}
$$

with the estimates

$$
\operatorname{deg}\left(A_{j} F_{j}\right) \leq \nu \operatorname{deg} G+n(D+|q|) .
$$

Proof. The proof is exactly similar to the proof of Theorem 4.1. At each point $\widetilde{Z} \in \mathbb{C}^{n+1} \backslash\{0\}$ such that $\left|Z_{0}\right| \leq \frac{1}{K}|\widetilde{Z}|$, it follows from (4.5) and the Briançon-Skoda theorem that

$$
\left(X_{0}^{n(|q|+D)}\right)_{\tilde{Z}} \in{ }^{\mathrm{h}} F_{1 n+1} \mathcal{O}_{\tilde{Z}}+\ldots+{ }^{\mathrm{h}} F_{k n+1} \mathcal{O}_{\tilde{Z}} .
$$

At each point $\widetilde{Z} \in \mathbb{C}^{n+1} \backslash\{0\}$ such that $\left|Z_{0}\right| \geq \frac{1}{2 K}\|\widetilde{Z}\|$,

$$
\left({ }^{\mathrm{h}} G^{\nu}\right)_{\tilde{Z}} \in{ }^{\mathrm{h}} F_{1 n+1} \mathcal{O}_{\tilde{Z}}+\ldots+{ }^{\mathrm{h}} F_{k n+1} \mathcal{O}_{\tilde{Z}} .
$$


So, at any point $\widetilde{Z} \in \mathbb{C}^{n+1} \backslash\{0\}$,

$$
\left(X_{0}^{n(|q|+D) \mathrm{h}} G^{\nu}\right)_{\tilde{Z}} \in{ }^{\mathrm{h}} F_{1 n+1} \mathcal{O}_{\tilde{Z}}+\ldots+{ }^{\mathrm{h}} F_{k n+1} \mathcal{O}_{\tilde{Z}} .
$$

Because of the hypothesis, it follows (as kindly pointed to us by Roger Gay) from [Ban], Corollary 4.3, p. 42 (which is in fact a variant of the Hartogs theorem), that (4.8) is also valid at $\widetilde{Z}=0$. So, once again

$$
X_{0}^{n(|q|+D) \mathrm{h}} G^{\nu}=\sum_{j=1}^{k} \widetilde{A}_{j}(\tilde{X})^{\mathrm{h}} F_{j}(\widetilde{X}) .
$$

Taking $X_{0}=1$ provides the result.

\section{References}

[Aiz] L. A. Aizenberg, Multidimensional logarithmic residues and their applications, in: Several Complex Variables II, Encyclopaedia Math. Sci. 8, Springer, 1994, 24-39.

[Ban] C. Banica and O. Stănăşila, Algebraic Methods in the Global Theory of Complex Spaces, Wiley, New York, 1976.

[BGVY] C. A. Berenstein, R. Gay, A. Vidras and A. Yger, Residue Currents and Bézout Identities, Progr. Math. 114, Birkhäuser, Basel, 1993.

[BY1] C. A. Berenstein and A. Yger, Effective Bézout identities in $\mathbb{Q}\left[z_{1}, \ldots, z_{n}\right]$, Acta Math. 166 (1991), 69-120.

[BY2] - - - Une formule de Jacobi et ses conséquences, Ann. Sci. École Norm. Sup. Paris 24 (1991), 363-377.

[Bie1] G. Biernat, On the sum of residues for a polynomial mapping, Bull. Soc. Sci. Lett. Łódź 40, Sér. Rech. Déform. 9 (1990), 73-83.

[Bie2] -, On the Jacobi-Kronecker formula for a polynomial mapping having zeroes at infinity, Bull. Soc. Sci. Lett. Łódź 42, Sér. Rech. Déform. 14 (1992/93), 103-111.

[BS] J. Briançon et H. Skoda, Sur la clôture intégrale d'un idéal de germes de fonctions holomorphes en un point de $\mathbb{C}^{n}$, C. R. Acad. Sci. Paris Sér. A Math. 278 (1974), 949-951.

[Br1] D. W. Brownawell, Bounds for the degrees in the Nullstellensatz, Ann. of Math. 126 (1987), 577-591.

[Br2] - A prime power version of the Nullstellensatz, preprint, 1989.

[CN] P. Cassou-Noguès, Quelques remarques sur les applications polynomiales propres, manuscript, 1994.

[CNPł] P. Cassou-Noguès et A. Płoski, Un théorème des zéros effectif, Bull. Polish Acad. Sci. 44 (1996), 61-70.

[CoS] D. Cox, J. Little and D. O'Shea, Ideals, Varieties and Algorithms, Undergrad. Texts Math., Springer, 1991.

[ElY] M. Elkadi and A. Yger, Residue currents and complexity problems, in: Topics in Complex Analysis, Banach Center Publ. 31, Inst. Math., Polish Acad. Sci., Warszawa, 1995, 173-186. 
[Fi] N. Fitchas et A. Galligo, Nullstellensatz effectif et conjecture de Serre (théorème de Quillen-Suslin) pour le Calcul Formel, Math. Nachr. 149 (1990), 231-253.

[HGi] M. Giusti, J. Heintz, J. E. Morais and L. M. Pardo, When polynomial equation systems can be "solved" fast, preprint, Laboratoire GAGE, École Polytechnique, 1995.

[GH] P. Griffiths and J. Harris, Principles of Algebraic Geometry, Wiley, New York, 1978.

[Je] Z. Jelonek, The set of points at which a polynomial map is not proper, Ann. Polon. Math. 48 (1993), 259-266.

[K] J. Kollár, Sharp effective Nullstellensatz, J. Amer. Math. Soc. 1 (1988), 963975.

[LT] J. Lipman and B. Teissier, Pseudo-rational local rings and a theorem of Briançon-Skoda, Michigan Math. J. 28 (1991), 97-116.

[Pe] O. Perron, Algebra I (Die Grundlagen), Göschens Lehrbücherei, Berlin und Leipzig, 1932.

[Pł1] A. Płoski, On the growth of proper polynomial mappings, Ann. Polon. Math. 45 (1985), 287-309.

[Pł2] -, Sur l'exposant d'une application analytique I, Bull. Polish Acad. Sci. Math. 32 (1984), 669-673.

[PłT] A. Płoski and P. Tworzewski, A separation condition for polynomial mappings, ibid. 44 (1996), to appear.

[T] B. Teissier, Résultats récents d'algèbre commutative effective, Séminaire Bourbaki, 1989/1990, exposé 718, Astérisque 189-190 (1990), 107-131.

[Y] A. Yger, Courants résidus et applications, Publications de l'Ecole Doctorale, Bordeaux, 1994.

Dipartimento di Matematica

Università della Calabria

Arcavacata di Rende (Cs), Italy

E-mail: fabiano@ccuws4.unical.it
Département de Mathématiques Université Bordeaux I 33405, Talence, France E-mail: yger@math.u-bordeaux.fr

Received on 7.3.1996

and in revised form on 4.7 .1996 